\title{
Spontaneous hemothorax caused by ruptured multiple mycotic aortic aneurysms: a case report and literature review
}

Po-Sung Li $i^{1}$, Chung-Lin Tsai ${ }^{2}$, Sung-Yuan Hu ${ }^{1,3,4,5,6,8^{*}}$, Tzu-Chieh Lin ${ }^{1,3,5,7}$ and Yao-Tien Chang ${ }^{1,3,5}$

\begin{abstract}
Background: Mycotic aortic aneurysm (MAA) is a rare clinical entity with an incidence of 1-3\%, but it is a life-threatening infection of aorta characterized by dilatation of aorta with false lumen. Multiple MAAs have been reported rarely with an incidence of $0.03 \%$ and associated with a high mortality rate of $80 \%$ if ruptured.

Case presentation: A hypertensive and diabetic 78-year-old man visited our emergency department complaining intermittent dull and tingled pain over the left flank region for 1 week. Chest X-ray showed left pleural effusion and hemothorax was confirmed by thoracocentesis. Computed tomography (CT) of chest demonstrated multiple thoracic aortic aneurysms and the pathological findings disclosed the diagnosis of multiple MAAs. He was discharged under an uneventful condition post-surgical aortic repair with adequate intravenous antibiotics for 4 weeks.
\end{abstract}

Conclusions: $C T$ scan may make a definite diagnosis of multiple MAAs and management with surgical debridement, aortic repair and full-course antibiotics for Gram-positive coccus and/or Gram-negative bacillus is recommended.

Keywords: Computed tomography (CT), Hemothorax, Mycotic aortic aneurysm (MAA)

\section{Background}

Hemothorax is the presence of blood in the pleural space. The source of blood may be trauma to the chest wall, lung parenchyma, heart, or great vessels, infection/ inflammation, malignancy, coagulopathy or congenital arteriovenous malformations. Mycotic aortic aneurysm (MAA) is a rare clinical entity with an incidence of $1-3 \%$, but it is a life-threatening infection of aorta characterized by dilatation of aorta with false lumen [1-5]. Multiple MAAs have been reported an incidence of $0.03 \%$ and associated with a high mortality rate of $70-80 \%$ if ruptured. Spontaneous hemothorax associated with ruptured MAAs has been reported rarely [6].

\footnotetext{
* Correspondence: song9168@pie.com.tw

'Department of Emergency Medicine, Taichung Veterans General Hospital, Taichung, Taiwan

${ }^{3}$ School of Medicine, Chung Shan Medical University, Taichung, Taiwan Full list of author information is available at the end of the article
}

\section{Case presentation}

A 78-year-old hypertensive and diabetic man had a history of coronary artery disease with percutaneous coronary intervention in 1997. Chest X-ray (Fig. 1a) was normal 11 months ago. He denied major chest trauma recently. He suffered from intermittent low grade fever, dull and tingled pain over left flank region and progressive dyspnea for 1 week. He was brought to our emergency department. On arrival, vital signs were a respiratory rate of 22 breaths per min, a heart rate of 125 beats per min, a blood pressure of $158 / 79 \mathrm{mmHg}$ and a body temperature of $36.6{ }^{\circ} \mathrm{C}$. Physical examination revealed a pale conjunctiva, no heart murmur, absent breathing sound of left lower lung, and knocking pain over left costovertebral angle. Significant laboratory evaluation revealed white blood cell counts (WBCs) of $13,700 / \mathrm{mm}^{3}$ with $76.3 \%$ of segmented neutrophils, hemoglobin of $7.8 \mathrm{~g} / \mathrm{dl}$, red blood cell counts (RBCs) of $4.26 \times 10^{6} / \mathrm{mm}^{3}$, platelet counts of $634 \times 10^{3} / \mathrm{mm}^{3}$, creatinine $1.5 \mathrm{mg} / \mathrm{dl}$, albumin $2.6 \mathrm{~g} / \mathrm{dl}$, protein $7.8 \mathrm{~g} / \mathrm{dl}$, lactate 


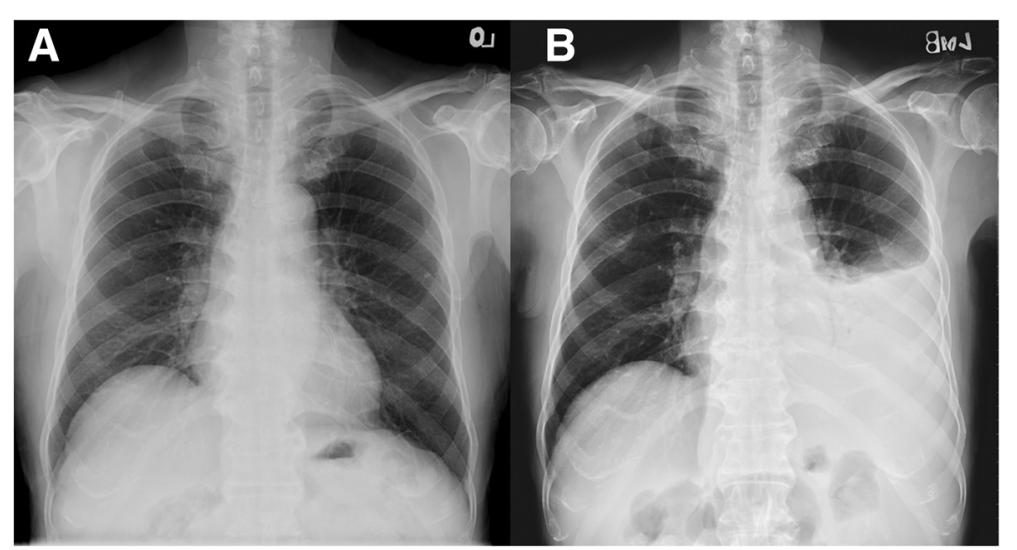

Fig. 1 Chest X-rays showed normal heart size, sharp cardiopleural angle, bare stent of coronary artery and no significant of aortic lesion (Panel a). Chest $\mathrm{X}$-rays revealed left pleural effusion and widening of upper descending aorta (Panel b)

dehydrogenase (LDH) 384 U/l, glucose of $257 \mathrm{mg} / \mathrm{dl}$, and high-sensitivity C-reactive protein (hs-CRP) of $18.6 \mathrm{mg} / \mathrm{dl}$. Chest X-ray showed left pleural effusion and mild widening of upper descending aorta (Fig. 1b). Blood-tinged pleural effusion was confirmed via a fine-needle aspiration under sono-guide after informed consent of patient. Analysis of pleural effusion were exudative and infection of pleural effusion should be considered, including WBCs of 1625/ $\mathrm{mm}^{3}$, with neutrophils of $62 \%$ and lymphocytes of $31 \%$, $\mathrm{RBCs}$ of $12,500 / \mathrm{mm}^{3}$, protein of $4.5 \mathrm{~g} / \mathrm{dl}, \mathrm{LDH}$ of $266 \mathrm{U} / \mathrm{l}$, glucose of $201 \mathrm{mg} / \mathrm{dl}$ and specific gravity of 1.030. Fever, flank pain, leukocytosis, elevated hs-CRP and hemothorax were clinical clues for high suspicion of ruptured infectious aorta. Computed tomography (CT) with intravenous contrast media depicted ruptured multiple thoracic aortic aneurysms at the level of between 8th and 11th thoracic spines with atelectasis of left lung and massive hemothorax (Fig. 2), so emergency surgical intervention with resection of fragile aorta, debridement of involved periaortic soft tissue, and reconstruction for descending aorta with Dacron graft of $20 \mathrm{~mm}$ was performed. Although the cultures of blood, pleural fluid, and resected aortic tissue showed no growth of bacteria or mycobacterium tuberculosis, the pathological findings demonstrated injury of vascular wall with acute and chronic inflammatory cells infiltration, fibrinous material coated on the internal luminal surface, focal abscess formation in vascular wall (Fig. 3) and no malignancy cells or granulation tissue. The serological screen for syphilis was non-reactive. The patient recovered gradually after surgical aortic repair, intensive care and adequate intravenous antibiotics for cover Gram-positive and Gramnegative bacteria with prostaphlin $2 \mathrm{~g}$ per $6 \mathrm{~h}$ and ceftriaxone $2 \mathrm{~g}$ per day for 4 weeks despite of negative-culture result. He was discharged under an uneventful condition with a regular follow-up of cardiovascular out-patient department for 2 years.

\section{Discussion}

Mycotic aortic aneurysm (MAA) is a rare but lifethreatening infection with an incidence of 1 3\% [1-5], characterized either by bacteremic seeding from extracardiac septicemia with bacterial invasion of an atherosclerotic vascular wall, endocarditis and contiguous/direct infection, leading to weakening and dilatation of vascular wall with formation of false lumen [5-7]. Multiple MAAs had been reported an incidence of $0.03 \%$, which had a high risk of

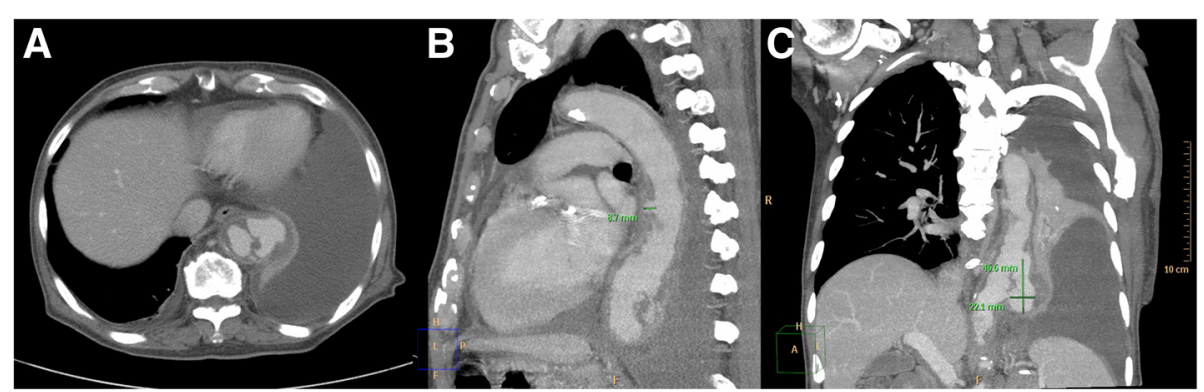

Fig. 2 Axial, sagittal and coronal views of computed tomographic scan with intravenous contrast media of chest showed irregular dumbbell-shaped aortic lesion (Panel a), multiple mushroom-like thoracic aortic aneurysms with the size of $8.7 \mathrm{~mm}$ in diameter (Panel b) and $46.6 \mathrm{~mm} \times 22.1 \mathrm{~mm}$ (Panel c) with periaortic soft tissue density at the level of between 8th and 11th thoracic spine, massive pleural effusion and atelectasis of left lung 


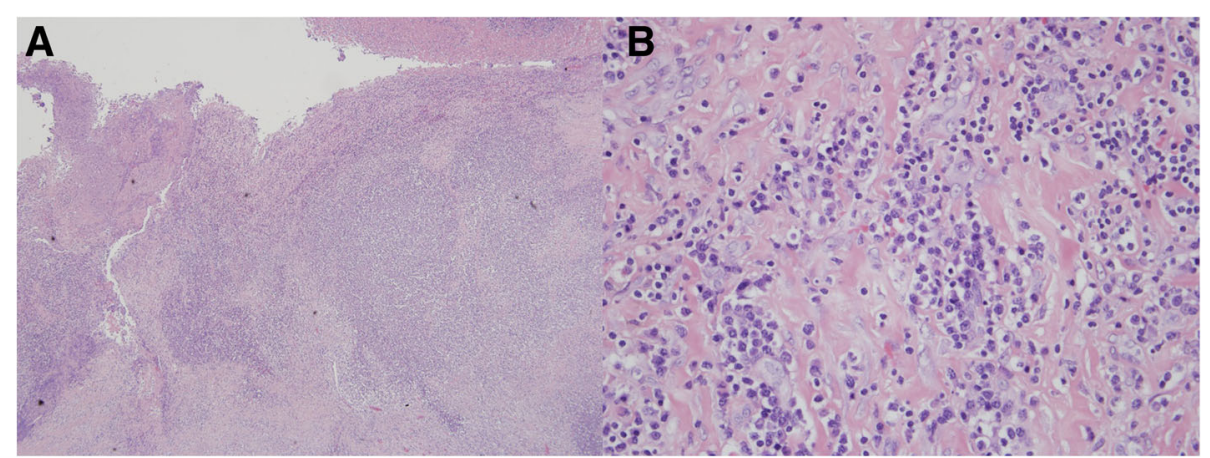

Fig. 3 Pathological findings disclosed fibrinous material coating on the internal luminal surface and formation of focal abscess was noted in the destructive vascular wall $(\times 100)$ (Panel a). There were infiltrations of acute and chronic inflammatory cells in the damaged vascular wall $(\times 1000)$ (Panel b)

rupture with a mortality rate of $80 \%$. Hemothorax as a sign of ruptured multiple MAAs was extremely rare and only few cases had been reported previously [6].

The differential diagnosis of hemothorax implicated the possibility of life-threatening underlying illnesses such as trauma, coagulopathy, spontaneous pneumothorax, vascular lesions, spontaneous esophageal rupture, infectious disease (such as pneumonia, tuberculous pleurisy, fungal disease, subphrenic abscess), malignancy, connective tissue disease (lupus pleuritis, rheumatoid pleurisy, Wegener's granulomatosis), pulmonary embolism, pulmonary sequestration, and endometriosis. They are difficulty to be differentiated from chest radiographs or clinical presentations. The rupture of thoracic mycotic aortic aneurysm (MAA) should be considered in the differential diagnosis of hemothorax [1-7].

MAA can develop either by infection of arterial wall or previous aneurysm with secondary infection. They maybe have led to a true or false lumen. Blood cultures are positive in $50-75 \%$ of MAA patients $[2,5,8]$. MAA is diagnosed according to clinical presentations with triad of fever, pain and pulsatile mass; laboratory investigations of leukocytosis and elevation of inflammatory biomarkers; radiological typical findings, including mushroom-like appearance, new aneurysm formation, rapid expansion or morphological change of known aneurysms, synchronous lesions, intramural or perivascular gas, edema, soft tissue mass or stranding, ring enhancement, disruption or disappearance of aortic calcification in late stage, and extravasation in rupture; cultures of blood and/or resected aortic tissue; and the histopathological features of an acute infection, including abscess and infiltration of neutrophils $[2,5,7]$.

Most common pathogens are bacteria such as Staphylococcus spp., Salmonella spp., and Treponema pallidum. The risk factor includes atherosclerosis, male sex, cigarette smoking, vascular abnormalities (pre-existing aneurysms), arterial trauma, old age, immunocompromised status, intravenous drug abusers, and infectious endocarditis. Medical treatment alone can't complete cure, so cardiovascular surgeons must perform surgical debridement and reconstruction of vascular continuity for thoracic aortic multiple MAAs plus intravenous antibiotics of 4-6 weeks at least for Gram-positive coccus and Gram-negative bacillus $[2,5,9-12]$.

The natural history of untreated mycotic aneurysms is of fatality from either massive hemorrhage or fulminant sepsis. Complications include $45 \%$ of rupture and $18 \%$ of fistula formation in late stage [5]. If an aortic mycotic aneurysm is diagnosed, surgical removal of an infected aneurysm must always be required because of a high mortality rate of $70-80 \%$ due to a high risk of rupture of mycotic aneurysm. Therefore undue delay of surgical intervention should be avoided. Emergency operation is recommended in those who have a ruptured aneurysm or are septic and unstable $[6,13]$. The optimal timing and best surgical procedure of surgical intervention are still difficult to be determined. The timing of surgical intervention should be determined by estimated risk of aneurysm rupture and surgical risk according to the patient's underlying condition and short-interval CT re-examinations $[13,14]$. The challenges of surgical intervention in patients with mycotic aneurysms are reinfection, difficulties of anastomosis lines in fragile cutend tissue, early and late postoperative bleeding, so a wide and extensive debridement of all infected tissue and a resection back to the healthy and non-infected wall of the aorta are mandatory $[15,16]$. In our case, he received emergency surgical repair immediately due to ruptured multiple MAAs after radiological and laboratory survey. He was fully recovered and received regular follow up at our hospital for 2 years.

\section{Conclusion}

Although the incidence is rare, the cause of spontaneous hemothorax should include rupture of MAA. Hold the 
implantation of chest tube if there is no trauma-related hemothorax. Etiologies of spontaneous hemothorax must be completed as soon as possible. Emergency surgical repair with adequate and full course of intravenous antibiotics are recommended if there is an evidence of rupture of MAA, such as hemothorax, because of high mortality. We recommend that the differential diagnosis of spontaneous hemothorax in high suspicion of infectious aorta should include MAA.

\section{Abbreviations}

CT: Computed tomography; LDH: Lactate dehydrogenase; MAAs: Mycotic aortic aneurysms; RBCs: Red blood cell counts; WBCs: White blood cell counts

\section{Acknowledgements}

Thanks for efforts of emergency resuscitation team, radiological technicians and division of infection in clinical diagnosis and management of this patient.

\section{Funding}

This research received no specific grant from any funding agency in the public, commercial, or not-for-profit sectors.

\section{Availability of data and materials}

Data sharing not applicable to this article as no datasets were generated or analysed during the current study.

\section{Authors' contributions}

The work presented here was carried out as collaboration among all the authors. Lin TC, Tsai CL, Li PS, Chang YT, and Hu SY were clinical responsible. Hu SY, Tsai CL and Lin TC defined the research theme. Lin TC, Chang YT, and Hu SY analyzed the data, interpreted the results and wrote the paper. Lin TC and Hu SY worked together on data collection and interpretation. Hu SY, Chang YT and Li PS co-designed the study and discussed the analysis, interpretation, and presentation. All authors have read and approved the final version of the manuscript.

\section{Ethics approval and consent to participate}

Ethical approval was not required, but written informed consent was obtained from the patient for publication of this case report and all accompanying images.

\section{Consent for publication}

Written informed consent was obtained from the patient for publication of this case report and accompanying images.

\section{Competing interests}

The authors declare that they have no competing interests.

\section{Publisher's Note}

Springer Nature remains neutral with regard to jurisdictional claims in published maps and institutional affiliations.

\footnotetext{
Author details

'Department of Emergency Medicine, Taichung Veterans General Hospital, Taichung, Taiwan. ${ }^{2}$ Division of Cardiac Surgery, Cardiovascular Center, Taichung Veterans General Hospital, Taichung, Taiwan. ${ }^{3}$ School of Medicine, Chung Shan Medical University, Taichung, Taiwan. ${ }^{4}$ Institute of Medicine, Chung Shan Medical University, Taichung, Taiwan. ${ }^{5}$ Department of Nursing, College of Health, National Taichung University of Science and Technology, Taichung, Taiwan. ${ }^{6}$ Department of Nursing, Central Taiwan Univeristy of Science and Technology, Taichung, Taiwan. ${ }^{7}$ College of Public Health, China Medical University, Taichung, Taiwan. ${ }^{8} 1650$ Taiwan Boulevard Sect. 4, Taichung 40705, Taiwan.
}

Received: 20 July 2017 Accepted: 16 November 2017

Published online: 02 December 2017

\section{References}

1. Voitle E, Hofmann W, Cejna M. Aortic emergencies-diagnosis and treatment - a pictorial review. Insights Imaging. 2015;6:17-32.

2. Stellmes A, Von Allmen $R$, Derungs $U$, et al. Thoracic endovascular aortic repair as emergency therapy despite suspected aortic infection. Interact Cardiovasc Thorac Surg. 2013;16:459-64.

3. Lee WK, Mossop PJ, Little AF, et al. Infected (mycotic) aneurysms: spectrum of imaging appearances and management. Radiographics. 2008;28:1853-68,

4. Kan CD, Lee HL, Yang YJ. Outcome after endovascular stent graft treatment for mycotic aortic aneurysm. J Vasc Surg. 2007;46:906-12.

5. Fisk M, Peck LF, Miyagi K, et al. Mycotic aneurysms: a case report, clinical review and novel imaging strategy. QJM. 2012;105:181-8.

6. Dwivedi AN, Srinivasan A, Jain S. Multiple Mycotic aneurysms of the abdominal aorta illustrated on MDCT scanner. J Clin Imaging Sci. 2015;5:49.

7. Abehsira G, Bagate F, Estagnasié P, Squara P. Multiple aortic mycotic aneurysms complicating a spondylitis without endocarditis. Intensive Care Med. 2013;39:1133.

8. Jaffer U, Gibbs R. Mycotic thoracoabdominal aneurysms. Ann Cardiothorac Surg. 2012;1:417-25

9. Nakashima M, Usui A, Oshima H, Ueda Y. The treatment of infectious aneurysms in the thoracic aorta; our experience in treating five consecutive patients. Interact Cardiovasc Thorac Surg. 2010;10:334-7.

10. Lopes RJ, Almeida J, Dias PJ, Pinho P, Maciel MJ. Infectious thoracic aortitis: a literature review. Clin Cardiol. 2009;32:488-90.

11. Brook I. Anaerobic bacteria as a cause of mycotic aneurysm of the aorta: microbiologyand antimicrobial therapy. Curr Cardiol Rev. 2009;5:36-9.

12. Hsu PJ, Lee CH, Lee FY, Liu JW. Clinical and microbiological characteristics of mycotic aneurysms in a medical center in southern Taiwan. J Microbiol Immunol Infect. 2008;41:318-24.

13. Kim YW. Infected aneurysm: current management. Ann Vasc Dis. 2010;3:7-15.

14. Usui A. Surgical management of infected thoracic aneurysms. Nagoya J Med Sci. 2013;75:161-7.

15. Dubois M, Daenens K, Houthoofd S, Peetermans WE, Fourneau I. Treatment of mycotic aneurysms with involvement of the abdominal aorta: single-centre experience in 44 consecutive cases. Eur J Vasc Endovasc Surg. 2010;40:450-6.

16. Huang YK, Chen CL, MS L, et al. Clinical, microbiologic, and outcome analysis of mycotic aortic aneurysm: the role of endovascular repair. Surg Infect. 2014;15:290-8.
Submit your next manuscript to BioMed Central and we will help you at every step:

- We accept pre-submission inquiries

- Our selector tool helps you to find the most relevant journal

- We provide round the clock customer support

- Convenient online submission

- Thorough peer review

- Inclusion in PubMed and all major indexing services

- Maximum visibility for your research

Submit your manuscript at www.biomedcentral.com/submit
) Biomed Central 